\title{
Computing the volume enclosed by a periodic surface and its variation to model a follower pressure
}

\author{
Mohammad Rahimi $^{1}$ · Kuan Zhang ${ }^{1} \cdot$ Marino Arroyo
}

Received: date / Accepted: date

\begin{abstract}
In modeling and numerically implementing a follower pressure in a geometrically nonlinear setting, one needs to compute the volume enclosed by a surface and its variation. For closed surfaces, the volume can be expressed as a surface integral invoking the divergence theorem. For periodic systems, widely used in computational physics and materials science, the enclosed volume calculation and its variation is more delicate and has not been examined before. Here, we develop simple expressions involving integrals on the surface, on its boundary lines, and point contributions. We consider two specific situations, a periodic tubular surface and a doubly periodic surface enclosing a volume with a nearby planar substrate, which are useful to model systems such as pressurized carbon nanotubes, supported lipid bilayers or graphene. We provide a set of numerical examples, which show that the familiar surface integral term alone leads to an incorrect volume evaluation and spurious forces at the periodic boundaries.
\end{abstract}

Keywords Periodic surface $\cdot$ Volume $\cdot$ Pressure $\cdot$ Follower load

\section{Introduction}

Thin shells and membranes often enclose a gas or a fluid, which exerts tractions on the thin material surface. Examples include air-bags, tires, submarine pipelines [22], pneumatic structures, hydroforming processes [1], lipid vesicles [10],

European Research Council (FP7/20072013)/ERC Grant Agreement no. 240487

K. Zhang and M. Arroyo

Universitat Politècnica de Catalunya-BarcelonaTech

E-mail: marino.arroyo@upc.edu

M. Rahimi

Princeton University

1. equal author contribution. or graphene membranes [6]. In equilibrium and depending on the system at hand, the effect of the enclosing medium can be modeled as a uniform pressure or by an enclosed volume constraint due to the incompressibility of the fluid inside. When the surface is pressurized with pressure $P$, a conservative and geometrically exact mechanical model can be formulated by including in the potential energy of the system the term $P V$, where $V$ is the enclosed volume. If instead the volume is constrained to $V_{0}$, then the potential energy can be augmented with the term $P\left(V-V_{0}\right)$ where $P$ is now a Lagrange multiplier that must be solved for. In either case, the pressure is a nonlinear force distribution that remains normal to the deformed surface-a follower load $[19,4,18]$. To derive the equilibrium equations or to compute the nodal pressure forces in a finite element method, it is necessary to compute the volume and its variation.

As elaborated in Section 2, for closed surfaces the divergence theorem allows us to compute the volume as a surface integral, and then obtain a simple expression for its variation. In the present paper, we consider periodic surfaces either under the action of a follower pressure or under an enclosed volume constraint. Periodic boundary conditions are useful in many applications in materials science, physics, or engineering, to model a representative cell within a larger and computationally intractable system. Examples include computational homogenization [11], or the analysis of cellular materials $[5,15]$. Periodic models are devoid of any undesirable artifact related with the boundary conditions used to truncate a very large domain, since the full model extended by periodicity does not have boundary. However, in these models the size of the periodic cell should be chosen carefully.

We show that the proper treatment of periodic surfaces requires computing, in addition to a surface integral, line integrals and a point contribution. We treat two cases: (1) periodic tubular surfaces and (2) doubly periodic surfaces 
enclosing a volume with a substrate. Our specific interest is in modeling pressurized carbon nanotubes [20] and supported graphene $[23,24]$ enclosing a fluid or a gas $[12,13$, $16]$, but the method is applicable to a wide range of problems such as supported lipid bilayers [21] or periodic structures in biomembrane tubes [7]. In the context of these applications, we provide numerical examples with Lagragian subdivision finite element calculations $[8,3]$, showing the correctness of the proposed method to compute the volume and its variation.

\section{Theory}

\subsection{Closed surfaces}

Consider a domain $\Omega$ with volume $V$ enclosed by a closed piecewise smooth surface $\Gamma=\partial \Omega \in \mathbb{R}^{3}$. The calculation of the volume can be recast into a surface integral by noting that $\operatorname{div} \boldsymbol{x}=3$ and invoking the divergence theorem

$V=\frac{1}{3} \int_{\Omega} \operatorname{div} x d V=\frac{1}{3} \int_{\Gamma} x \cdot n d S$,

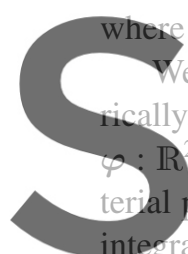

$n$ is the outward normal to consider now that the surfact , e.g. by Lagragian coordinates,

$\mathbb{R}^{2} \supset \Gamma \rightarrow \Gamma \subset \mathbb{R}^{3}$ such that particles. The surface integ

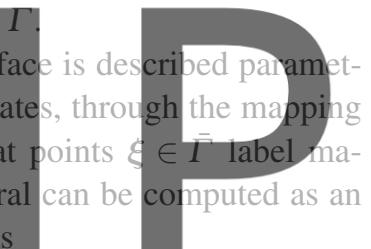

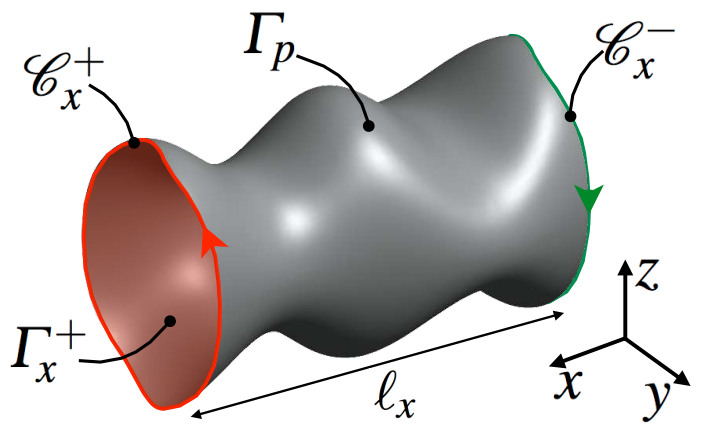

Fig. 1 Sketch of a tubular periodic surface $\Gamma_{p}$, highlighting its boundary curves $\mathscr{C}_{x}^{+}$and $\mathscr{C}_{x}^{-}$, and one of the lateral bounding surfaces, $\Gamma_{x}^{+}$.

can be easily extended to piecewise smooth surfaces [2]. Consider the following integral

$I=\frac{1}{3} \int_{\partial \Gamma} \delta x \cdot(x \times d \ell)=\frac{1}{3} \int_{\partial \bar{\Gamma}} w \cdot \tau d \bar{\ell}$

where $w=\left\{\delta \varphi \cdot\left(\varphi \times g_{1}\right), \delta \varphi \cdot\left(\varphi \times g_{2}\right)\right\}$, $\tau$ is a unit tangent vector to the curve $\partial \bar{\Gamma}$ consistent with the orientation given by the normal, and $d \bar{\ell}$ is the line element in the reference space. To obtain the right-hand side, we have expressed $d \ell=\left(\tau^{1} g_{1}+\tau^{2} g_{2}\right) d \bar{\ell}$ to perform the change of variables. In-
voking Stokes's theorem in the plane and recalling the defi-
nition of $w$, this integral can be written as
$I=\frac{1}{3} \int_{\bar{\Gamma}}\left(\partial w_{2} / \partial \xi^{1}-\partial w_{1} / \partial \xi^{2}\right) d \xi_{1} d \xi_{2}$
$=\frac{1}{3} \int_{I}\left[\delta g_{1} \cdot\left(\varphi \times g_{2}\right)+\delta \varphi \cdot\left(g_{1} \times g_{2}\right)\right.$ $=\frac{1}{3} \int_{\bar{\Gamma}}\left[\delta g_{1} \cdot\left(\varphi \times g_{2}\right)+\delta \varphi \cdot\left(g_{1} \times g_{2}\right)\right.$

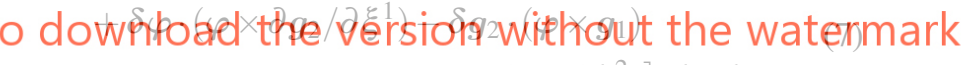
$\left.-\delta \varphi \cdot\left(g_{2} \times g_{1}\right)-\delta \varphi \cdot\left(\varphi \times \partial g_{1} / \partial \xi^{2}\right)\right] d \xi_{1} d \xi_{2}$

where $g_{\alpha}=\partial \varphi / \partial \xi^{\alpha}$ with $\alpha=1,2$. Noting that $n \circ \varphi=$ $\boldsymbol{g}_{1} \times \boldsymbol{g}_{2} /\left|\boldsymbol{g}_{1} \times \boldsymbol{g}_{2}\right|$, we finally obtain

$V=\frac{1}{3} \int_{\bar{\Gamma}} \boldsymbol{\varphi} \cdot\left(\boldsymbol{g}_{1} \times \boldsymbol{g}_{2}\right) d \xi_{1} d \xi_{2}$

The variation of the volume is then

$$
\begin{aligned}
\delta V= & \frac{1}{3} \int_{\bar{\Gamma}}\left[\delta \boldsymbol{\varphi} \cdot\left(\boldsymbol{g}_{1} \times \boldsymbol{g}_{2}\right)\right. \\
& \left.+\boldsymbol{\varphi} \cdot\left(\delta \boldsymbol{g}_{1} \times \boldsymbol{g}_{2}+\boldsymbol{g}_{1} \times \delta \boldsymbol{g}_{2}\right)\right] d \xi_{1} d \xi_{2}
\end{aligned}
$$

where $\delta g_{\alpha}=\partial(\delta \varphi) / \partial \xi^{\alpha}$. This expression can be further simplified to the usual expression $[4,19]$

$\delta V=\int_{\Gamma} \boldsymbol{\delta} \boldsymbol{x} \cdot \boldsymbol{n} d S=\int_{\bar{\Gamma}} \boldsymbol{\delta} \boldsymbol{\varphi} \cdot\left(\boldsymbol{g}_{1} \times \boldsymbol{g}_{2}\right) d \xi_{1} d \xi_{2}$.

Since a derivation of the last step from Eq. (4) to Eq. (5), which shows its compatibility with Eq. (1), is rarely presented in the literature, we provide it next. This derivation is then extended to treat the periodic case. We develop next a direct parametric calculation assuming $\Gamma$ is smooth, which

$$
\begin{aligned}
= & \frac{1}{3} \int_{\bar{\Gamma}}\left[2 \boldsymbol{\delta} \boldsymbol{\varphi} \cdot\left(\boldsymbol{g}_{1} \times \boldsymbol{g}_{2}\right)\right. \\
& \left.-\boldsymbol{\varphi} \cdot\left(\boldsymbol{\delta} \boldsymbol{g}_{1} \times \boldsymbol{g}_{2}+\boldsymbol{g}_{1} \times \delta \boldsymbol{g}_{2}\right)\right] d \xi_{1} d \xi_{2},
\end{aligned}
$$

where we have used the smoothness of the surface in assuming that $\partial \boldsymbol{g}_{2} / \partial \xi^{1}=\partial \boldsymbol{g}_{1} / \partial \xi^{2}$. Now, adding the previous equation and Eq. (4), we obtain

$\delta V+I=\int_{\bar{\Gamma}} \delta \varphi \cdot\left(\boldsymbol{g}_{1} \times \boldsymbol{g}_{2}\right) d \xi_{1} d \xi_{2}$

Obviously, for a closed surface $\partial \Gamma=\emptyset$ and $I=0$, and hence we recover the expected result in Eq. (5). An alternative derivation can be obtained by taking variations directly to the right-hand side of Eq. (1) and resorting to differential geometry identities [9].

\subsection{Tubular periodic surfaces}

Consider now a tubular periodic surface along the $x$ axis, $\Gamma_{p}$, with periodicity length $\ell_{x}$. We denote the boundary closed 
curves of $\Gamma_{p}$ by $\mathscr{C}_{x}^{+}$and $\mathscr{C}_{x}^{-}$, which are periodic images of each other and therefore if $\boldsymbol{x} \in \mathscr{C}_{x}^{-}$then $\boldsymbol{x}+\ell_{x} \boldsymbol{e}_{x} \in \mathscr{C}_{x}^{+}$, where $e_{x}$ is the unit basis vector along $x$. Note that for Lagrangian surfaces, in general the curve $\mathscr{C}_{x}^{+}$does not lie on a plane. See Figure 1 for an illustration.

The surface extended by periodicity encloses an infinite volume, but a periodic domain laterally enclosed by $\Gamma_{p}$ still defines an unambiguous volume of the periodic cell. Consider the closed surface $\Gamma=\Gamma_{p} \cup \Gamma_{x}^{+} \cup \Gamma_{x}^{-}$, where $\Gamma_{x}^{+}$is any smooth surface having $\mathscr{C}_{x}^{+}$as its boundary and $\Gamma_{x}^{-}$its periodic image.

The volume of the periodic cell is then

$$
\begin{aligned}
3 V & =\int_{\Gamma_{p}} \boldsymbol{x} \cdot \boldsymbol{n} d S+\int_{\Gamma_{x}^{+}} \boldsymbol{x} \cdot \boldsymbol{n} d S+\int_{\Gamma_{x}^{-}} \boldsymbol{x} \cdot \boldsymbol{n} d S \\
& =\int_{\Gamma_{p}} x \cdot n d S+\ell_{x} \int_{\Gamma_{x}^{+}} e_{x} \cdot n d S
\end{aligned}
$$

where in the last equality we have used the fact that $\Gamma_{x}^{-}$is the periodic image of $\Gamma_{x}^{+}$with opposite outward normal. Noting that $\boldsymbol{e}_{x}=$ curl $\boldsymbol{q}_{x}$ where $\boldsymbol{q}_{x}=1 / 2\{0,-z, y\}^{T}$ and using Stokes' theorem, we obtain

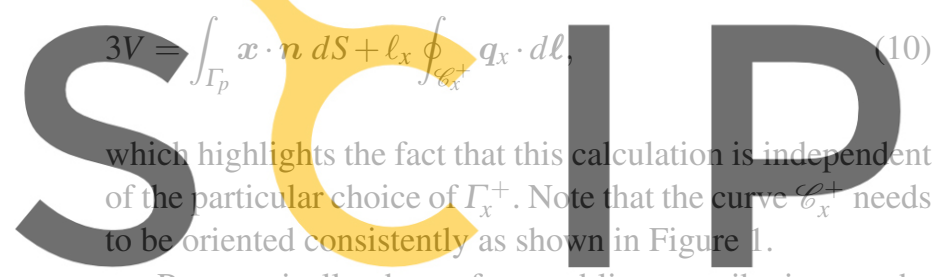

Parametrically, the surface and line contributions to the volume calculation take the form

Register for free at https//www.scipedia.com

$$
\begin{aligned}
3 V & =\int_{\overline{\bar{\Gamma}}_{p}} \boldsymbol{\varphi} \cdot\left(g_{1} \times g_{2}\right) d \xi_{1} d \xi_{2}+\frac{\ell_{x}}{2} \int_{\overline{\bar{C}}}\left\{0,-c_{z}, c_{y}\right\} \cdot t d \eta \\
& =\int_{\bar{\Gamma}_{p}} \boldsymbol{\varphi} \cdot\left(\boldsymbol{g}_{1} \times \boldsymbol{g}_{2}\right) d \xi_{1} d \xi_{2}+\frac{\ell_{x}}{2} \int_{\overline{\mathscr{C}}} \boldsymbol{e}_{x} \cdot(\boldsymbol{c} \times \boldsymbol{t}) d \eta,
\end{aligned}
$$

where now $\varphi$ parametrizes the tubular periodic surface $\Gamma_{p}$, $c: \mathbb{R} \supset \overline{\mathscr{C}} \rightarrow \mathscr{C}_{x}^{+} \subset \mathbb{R}^{3}$ parametrizes the closed curve (with the orientation induced by the normal), and $t=\partial c / \partial \eta$ is its tangential vector. In fact, $c(\eta)$ is the restriction of $\varphi\left(\xi^{1}, \xi^{2}\right)$ to the boundary curves of $\bar{\Gamma}_{p}$. The variation of the volume is then

$$
\begin{aligned}
3 \delta V= & \int_{\bar{\Gamma}_{p}}\left[\delta \boldsymbol{\varphi} \cdot\left(\boldsymbol{g}_{1} \times \boldsymbol{g}_{2}\right)\right. \\
& \left.+\boldsymbol{\varphi} \cdot\left(\boldsymbol{\delta} \boldsymbol{g}_{1} \times \boldsymbol{g}_{2}+\boldsymbol{g}_{1} \times \delta \boldsymbol{g}_{2}\right)\right] d \xi_{1} d \xi_{2} \\
& +\frac{\ell_{x}}{2} \int_{\overline{\mathscr{C}}} \boldsymbol{e}_{x} \cdot(\boldsymbol{\delta} \boldsymbol{c} \times \boldsymbol{t}+\boldsymbol{c} \times \delta \boldsymbol{t}) d \eta
\end{aligned}
$$

Recalling the derivation in Section 2.1, adapting Eq. (8) to the present situation, and using periodicity, we can further simplify the surface integral as follows

$$
\begin{aligned}
\delta V= & \int_{\bar{\Gamma}_{p}} \boldsymbol{\delta} \boldsymbol{\varphi} \cdot\left(\boldsymbol{g}_{1} \times \boldsymbol{g}_{2}\right) d \xi_{1} d \xi_{2} \\
& -\frac{1}{3} \int_{\mathscr{C}_{x}^{+}} \boldsymbol{\delta} \boldsymbol{x} \cdot(\boldsymbol{x} \times d \boldsymbol{\ell})-\frac{1}{3} \int_{\mathscr{C}_{x}^{-}} \boldsymbol{\delta} \boldsymbol{x} \cdot(\boldsymbol{x} \times d \boldsymbol{\ell}) \\
& +\frac{\ell_{x}}{6} \int_{\overline{\mathscr{C}}} \boldsymbol{e}_{x} \cdot(\boldsymbol{\delta} \boldsymbol{c} \times \boldsymbol{t}+\boldsymbol{c} \times \boldsymbol{\delta} \boldsymbol{t}) d \eta \\
= & \int_{\bar{\Gamma}_{p}} \boldsymbol{\delta} \boldsymbol{\varphi} \cdot\left(\boldsymbol{g}_{1} \times \boldsymbol{g}_{2}\right) d \xi_{1} d \xi_{2} \\
& -\frac{\ell_{x}}{3} \int_{\overline{\mathscr{C}}} \boldsymbol{\delta} \boldsymbol{c} \cdot\left(\boldsymbol{e}_{x} \times \boldsymbol{t}\right) d \eta \\
& +\frac{\ell_{x}}{6} \int_{\overline{\mathscr{C}}} \boldsymbol{e}_{x} \cdot(\boldsymbol{\delta} \boldsymbol{c} \times \boldsymbol{t}+\boldsymbol{c} \times \boldsymbol{\delta} \boldsymbol{t}) d \eta \\
= & \int_{\bar{\Gamma}_{p}} \boldsymbol{\delta} \boldsymbol{\varphi} \cdot\left(\boldsymbol{g}_{1} \times \boldsymbol{g}_{2}\right) d \xi_{1} d \xi_{2} \\
& +\frac{\ell_{x}}{6} \int_{\overline{\mathscr{C}}} e_{x} \cdot(3 \delta c \times t+c \times \delta t) d \eta
\end{aligned}
$$

As we show later, the line contribution is essential to properly compute the volume and its variation. Without this term, the nodal pressures experience a spurious boundary effect.

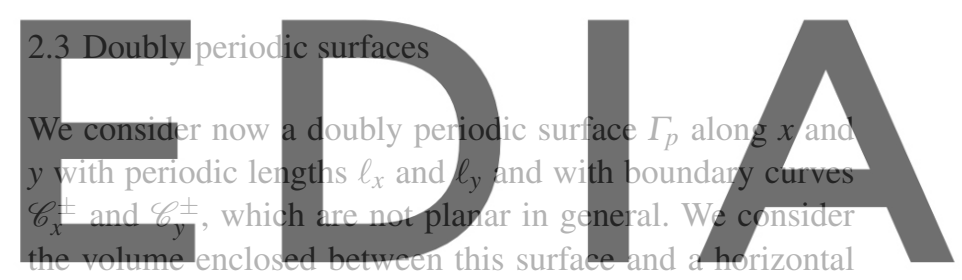

plane, which without loss of generality, is given by $z=0$.

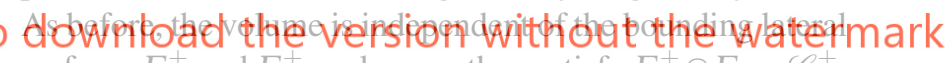
surfaces $\Gamma_{x}^{ \pm}$and $\Gamma_{y}^{ \pm}$, as long as they satisfy $\Gamma_{x}^{ \pm} \cap \Gamma_{p}=\mathscr{C}_{x}^{ \pm}$, $\Gamma_{y}^{ \pm} \cap \Gamma_{p}=\mathscr{C}_{y}^{ \pm}$and the periodicity requirements. We denote by $\Gamma_{z=0}$ the bottom bounding surface.

Without loss of generality, we choose for convenience $\Gamma_{x}^{ \pm}$and $\Gamma_{y}^{ \pm}$such that $\Gamma_{x}^{ \pm} \cap \Gamma_{y}^{ \pm}$is a vertical segment delimited by the corner point $Q=\left(Q_{x}, Q_{y}, Q_{z}\right)$ (or one of its four periodic images) and the plane $z=0$. Furthermore, we choose $\Gamma_{x}^{ \pm}$and $\Gamma_{y}^{ \pm}$such that $\mathscr{S}_{x}^{ \pm}=\Gamma_{x}^{ \pm} \cap \Gamma_{z=0}$ or $\mathscr{S}_{y}^{ \pm}=$ $\Gamma_{y}^{ \pm} \cap \Gamma_{z=0}$ are line segments aligned with the $y$ and $x$ axes respectively (see Figure 2 for an illustration). Since $\Gamma_{p} \cup \Gamma_{x}^{+} \cup$ $\Gamma_{x}^{-} \cup \Gamma_{y}^{+} \cup \Gamma_{y}^{-} \cup \Gamma_{z=0}$ is a closed piecewise smooth surface and the intersection of any two of these surfaces is a set of zero measure, we have

$3 V=\int_{\Gamma_{p}} \boldsymbol{x} \cdot \boldsymbol{n} d S+\ell_{x} \int_{\Gamma_{x}^{+}} \boldsymbol{e}_{x} \cdot \boldsymbol{n} d S+\ell_{y} \int_{\Gamma_{y}^{+}} \boldsymbol{e}_{y} \cdot \boldsymbol{n} d S$,

where we have used the same argument as in Eq. (9) along $x$ and $y$, and the fact that $\boldsymbol{x} \cdot \boldsymbol{n}=0$ on $\Gamma_{z=0}$. Noting that $\boldsymbol{e}_{y}=$ curl $\boldsymbol{q}_{y}$ where $\boldsymbol{q}_{y}=1 / 2\{z, 0,-x\}^{T}$ and invoking Stokes' theorem, we have

$3 V=\int_{\Gamma_{p}} \boldsymbol{x} \cdot \boldsymbol{n} d S+\ell_{x} \oint_{\partial \Gamma_{x}^{+}} \boldsymbol{q}_{x} \cdot d \boldsymbol{\ell}+\ell_{y} \oint_{\partial \Gamma_{y}^{+}} \boldsymbol{q}_{y} \cdot d \boldsymbol{\ell}$. 


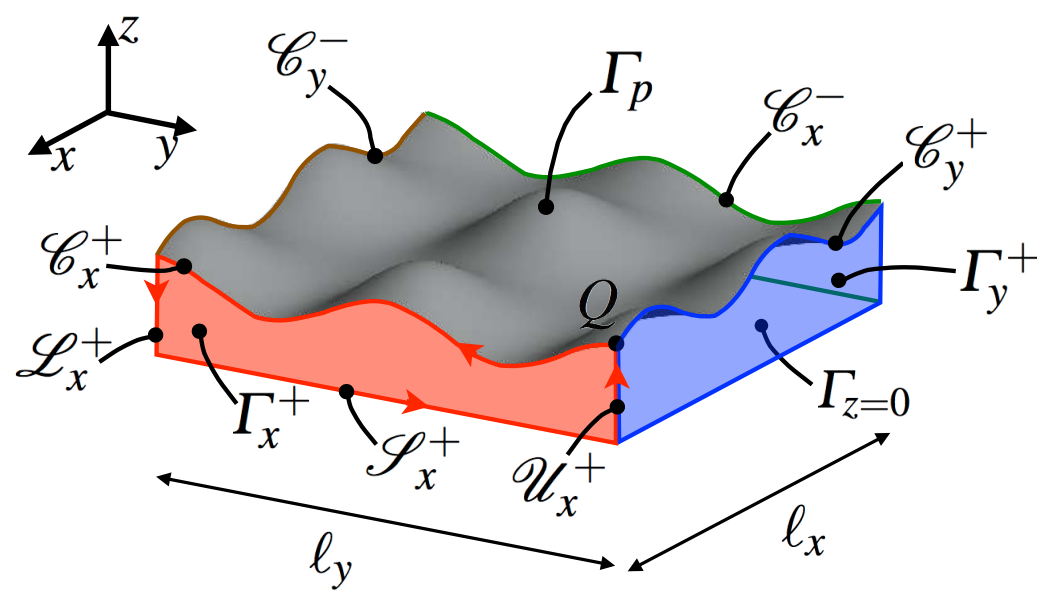

Fig. 2 Sketch of a doubly periodic surface $\Gamma_{p}$ enclosing a volume with a substrate located at $z=0$. As noted in the text, the calculation of the volume is independent of the particular choice of lateral bounding surfaces, as long as they satisfy the periodicity reguirements.

Focusing on the second term, noting that $\partial \Gamma_{x}^{+}=\mathscr{C}_{x}^{+} \cup \mathscr{L}_{x}^{+} \cup$ $\mathscr{S}_{x}^{+} \cup \mathscr{U}_{x}^{+}$as illustrated in in Figure 2, and recalling that $\mathscr{L}_{x}^{+}$and $\mathscr{U}_{x}^{+}$are straight segments aligned with the $z$ axis and $\mathscr{S}_{x}^{+}$is aligned with the $y$ axis, we have

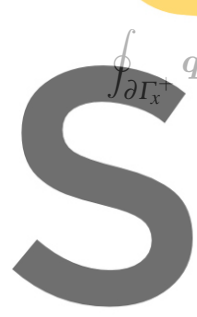
(a)

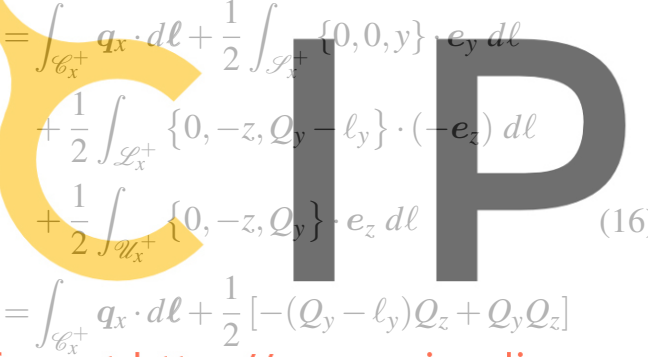

\section{Register for free at https/, $q_{2}$ www.scipedia.com to}

Repeating the same argument with the third term in Eq. (15), we obtain a formula for the volume including a surface contribution, two curve contributions, and a point contribution

$3 V=\int_{\Gamma_{p}} \boldsymbol{x} \cdot \boldsymbol{n} d S+\int_{\mathscr{C}_{x}^{+}} \boldsymbol{q}_{x} \cdot d \boldsymbol{\ell}+\int_{\mathscr{C}_{y}^{+}} \boldsymbol{q}_{y} \cdot d \boldsymbol{\ell}+\ell_{x} \ell_{y} Q_{z}$.

We can express this calculation parametrically in Lagrangian coordinates as

$$
\begin{aligned}
3 V & =\int_{\bar{\Gamma}_{p}} \boldsymbol{\varphi} \cdot\left(\boldsymbol{g}_{1} \times \boldsymbol{g}_{2}\right) d \xi_{1} d \xi_{2}+\ell_{x} \ell_{y} Q_{z} \\
& +\frac{\ell_{x}}{2} \int_{\overline{\mathscr{C}}_{x}} \boldsymbol{e}_{x} \cdot\left(\boldsymbol{c}_{x} \times \boldsymbol{t}_{x}\right) d \eta+\frac{\ell_{y}}{2} \int_{\overline{\mathscr{C}}_{y}} \boldsymbol{e}_{y} \cdot\left(\boldsymbol{c}_{y} \times \boldsymbol{t}_{y}\right) d \eta
\end{aligned}
$$

where $c_{x}$ and $c_{y}$ are restrictions of $\boldsymbol{\varphi}$ parametrizing the boundary curves $\mathscr{C}_{x}^{+}$and $\mathscr{C}_{y}^{+}$respectively, such that $\boldsymbol{c}_{x}\left(\overline{\mathscr{C}}_{x}\right)=\mathscr{C}_{x}^{+}$ and $\boldsymbol{c}_{y}\left(\overline{\mathscr{C}}_{y}\right)=\mathscr{C}_{y}^{+}, \boldsymbol{t}_{x}=\partial \boldsymbol{c}_{x} / \partial \eta$ and $\boldsymbol{t}_{y}=\partial \boldsymbol{c}_{y} / \partial \eta$. Analogously to Eq. (13), we can compute the variation of the

\section{volume as}
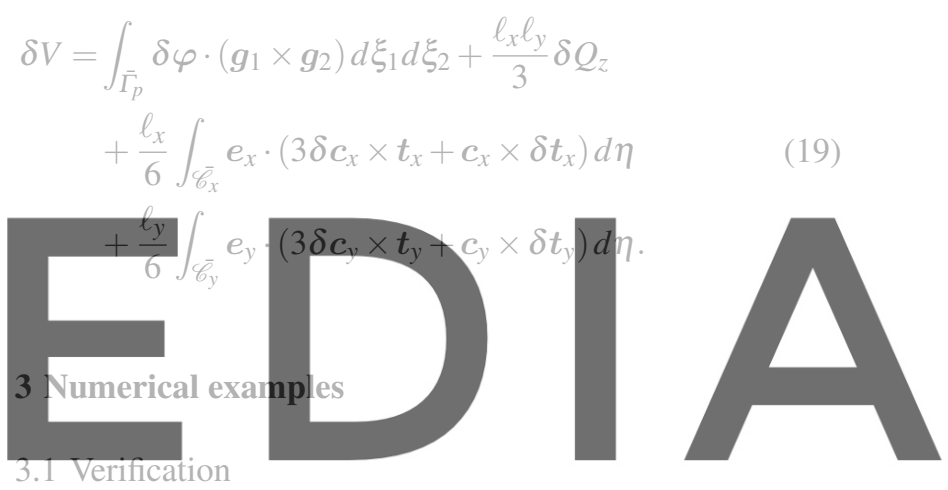

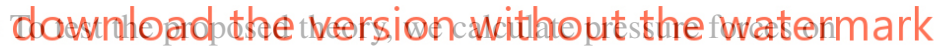 a tubular pressurized surface. The surface is discretized with} subdivision finite elements [8], the expressions in the previous section are implemented in the finite element framework, and the nodal pressure forces are computed. If the surface is described with a Lagrangian finite element mesh, $\varphi$ is a piecewise mapping defined on the elements, and the integrals in resulting expressions for $V$ and $\delta V$ can be computed element-by-element in the reference element. The pressure finite element nodal forces follow from $P \delta V$ by taking $\delta \varphi$ proportional to the basis functions.

Since the mesh is nearly uniform, we expect the pressure forces to be nearly identical on every node and normal to the surface. Furthermore, since the boundary of the periodic surface is fictitious, the pressure forces should be completely oblivious to the mesh free edges. Figure 3(a) shows that the pressure forces computed with just the surface contribution as in Eq. (5), i.e. ignoring the line contribution in Eq. (13), results in spurious forces at the boundary of the finite element mesh. Note that, because the support of the subdivision basis functions spans two rings of triangles, the spurious forces affect two rows of nodes close to the boundary. As shown in Fig. 3(b), the line term corrects this incon- 


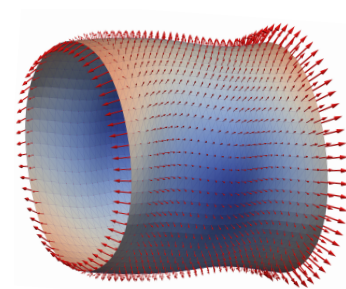

$\mathrm{a}$

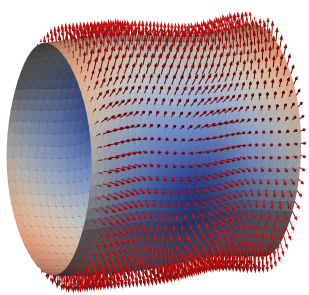

$\mathrm{b}$
Fig. 3 Nodal forces resulting from a follower pressure on a tubular periodic surface discretized with subdivision finite elements. The color map represents the deviation from a cylindrical surface. In (a), unphysical forces appear near the boundaries of the computational domain. In contrast, (b) shows the correct distribution of nodal forces on the tube. The nodal forces have been scaled differently.

sistency, and results in uniform forces, normal to the surface, which are unaware of the periodic boundary

Figure 4(a,b) shows an analogous study for a doubly periodic surface. In this case, in addition of spurious boundary forces, spurious corner forces appear in only the surface contribution to the variation of the volume is accounted for. The correct nodal forces are recovered by including the line and point contributions in Eq. (19).

In addition to these visual tests, we performed system-

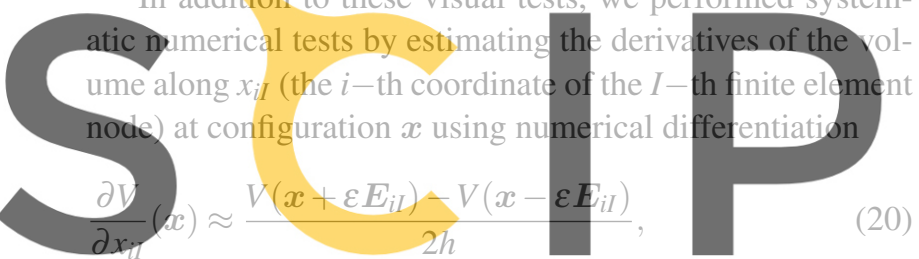

where $\varepsilon=10^{-6}$ is the magnitude of a small perturbation

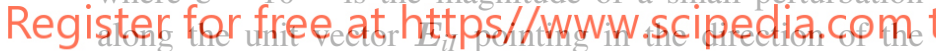

$i$-th coordinate of the $I$-th node. This estimation should be very close to the analytical calculation of the derivative provided by $\delta V\left[N_{I} e_{i}\right]$, where $N_{I}$ is the basis function of the $I$-th node and $e_{i}$ are the Cartesian basis vectors. Figure 4(c) shows the nodal vector field obtained with numerical differentiation. It is clearly very similar to the analytical calculation shown in Fig. 4(b). In fact, the norm of the difference between the analytical and the numerical differentiation estimate is $1.98 \times 10^{-8}$.

\subsection{Application to supported graphene}

As an application of the theory, we perform a simulation of a supported graphene sheet adhered to the substrate upon biaxial compression and under internal pressure in graphenesubstrate interstitial space [24]. Graphene is modeled as an elastic sheet using an atomistic-based continuum model within a geometrically exact framework, which adheres to the substrate with a continuum version of a Lennard-Jones potential and experiences a frictional sliding forces. Since graphene is impermeable to gases, we model the presence of gas molecules
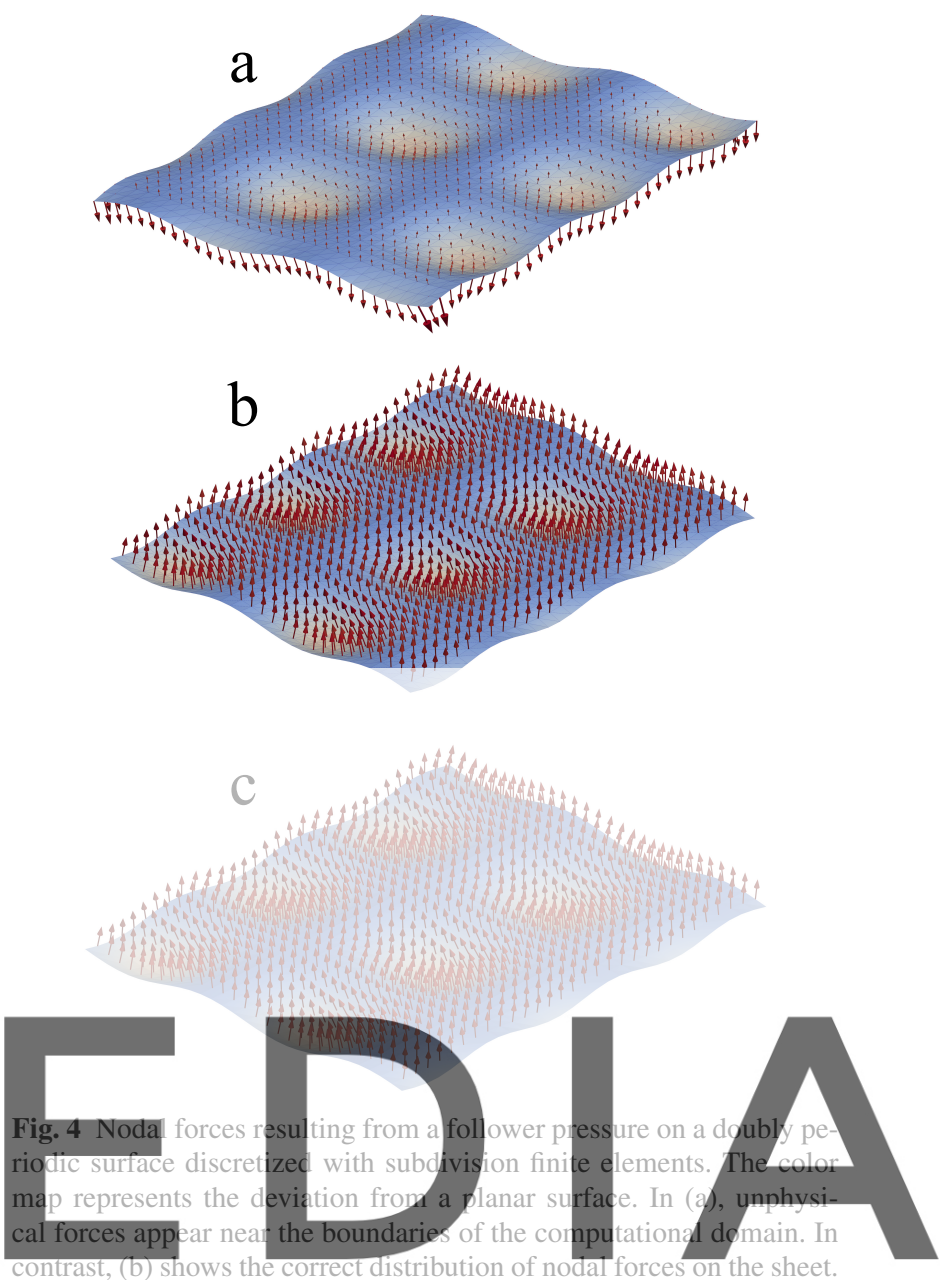

(c) Shows the distribution of nodal forces estimated using numerical

\section{differwintion a se tpine teversion without the watermark}

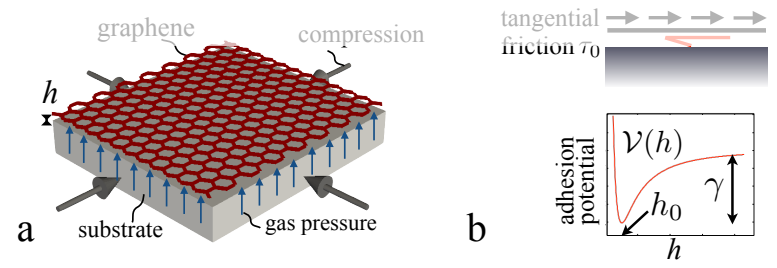

Fig. 5 (a) Model setup for a periodic graphene sample supported on a substrate, laterally compressed, and subject to the interstitial pressure caused by gas molecules trapped between the substrate and graphene. (b) Graphene interacts with the substrate through an adhesion potential $\mathscr{V}(h)$ characterized by the adhesion energy $\gamma$ and the equilibrium separation $h_{0}$, and through tangential forces opposing sliding, modeled as dry friction with interfacial shear strength $\tau_{0}$.

trapped in the interstitial space using the ideal gas law, by which the pressure difference across the membrane follows from $p=n R T / V$, where $n$ is the number of moles of gas trapped underneath the membrane, $R$ is the ideal gas constant, $T$ is the absolute temperature. We introduce this effect by adding the term $-n R T \ln \left(V / V_{0}\right)$ to the free-energy of the system, where $V_{0}$ is an arbitrary reference volume. The 


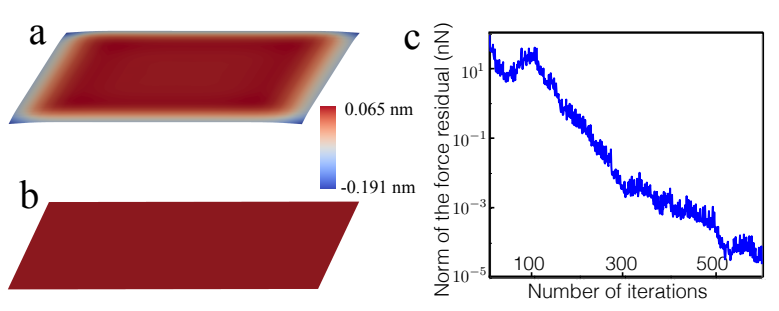

Fig. 6 Periodic graphene sample placed in contact with the substrate under interstitial pressure, and relaxed by minimizing the energy using a quasi-Newton algorithm. (a) shows the resulting shape when the boundary and corner contributions in the volume variation are ignored, exhibiting spurious deformations at the fictitious boundary of the periodic domain. (b) shows the relaxed shape using the correct calculation of the volume and its variation. The solution is planar as physically expected. The color map describes the out-of-plane displacement relative to the equilibrium separation of graphene $h_{0}$. (c) shows the convergence of the minimization algorithm in the case of (b) in terms of the norm of the force residual.

resulting nodal forces then follow as $(-n R T / V) \delta V\left[N_{I} e_{i}\right]$. Because of geometric nonlinearity, the pressure difference across the graphene sheet is a follower load. To obtain equilibrium configurations, we minimize the total free energy of the system using a quasi-Newton method with line-search,

which evaluates the energy and its gradient with respect to the rodal degrees of freedom. See

of the model and a motivation of the

an illustration.

first place a periodic graphene sample on a substrate
ubject it to interstitial pressure but no strain. For a mod-

erate number of trapped molecules, we expect that the solu-

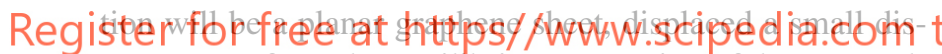
tance away from the equilibrium separation of the LennardJones adhesion potential, $h_{0}$. Figure 6(a) shows that, when the boundary and corner terms of the volume and its variation are ignored, the result after the quasi-Newton iteration exhibits spurious deformations at the boundary of the periodic domain. In contrast, the correct calculation of the volume and its variation results in a planar state as expected, see Fig. 6(b). The numerical convergence of the iterative method is shown in Fig. 6(c).

We then consider a more challenging calculation, where a $500 \mathrm{~nm} \times 500 \mathrm{~nm}$ graphene sample is first biaxially compressed. Graphene is easily bendable but very hard to stretch, leading to complex buckling patterns. Fig. 7(a-c) shows the morphological evolution from randomly distributed and small rippling to a localized wrinkling network, as often observed in supported graphene grown by chemical vapor deposition $[14,17]$. In the second stage, we examine the behavior of the system as the number of trapped molecules increases. During this process, the wrinkle network is simplified (Fig. 7(d)), and wrinkles thicken Fig. 7(e), suggesting these mechanically self-assembled networks could materialize nano-fluidic channels. With a high internal pressure, some wrinkles dra-
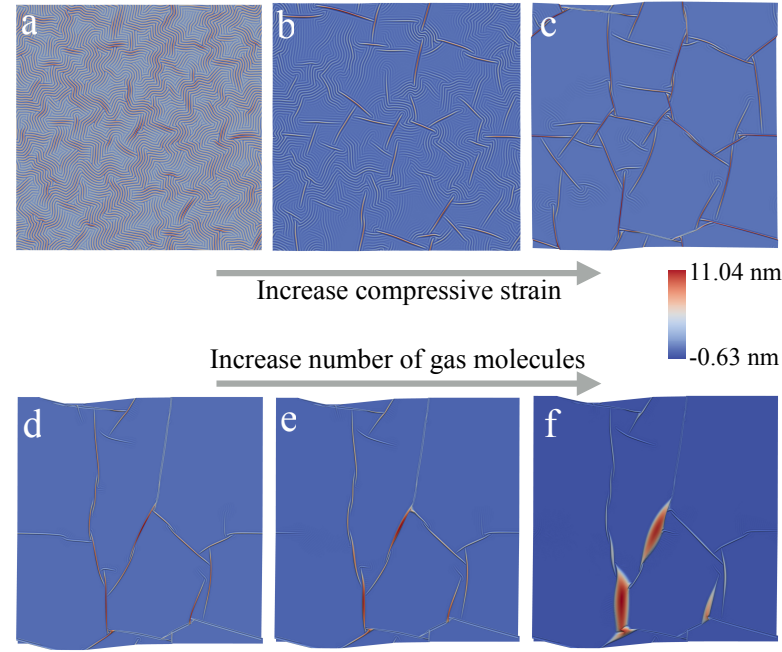

Fig. 7 Spontaneous morphological transitions of supported graphene upon biaxial compression and internal pressure. In the first stage under biaxial strain, (a) Uniformly distributed ripples; (b) nucleation of short wrinkles; (c) wrinkling network. In the second stage, by increasing the internal pressure of interstitial space while maintaining the biaxial strain constant, the wrinkling network is reorganized and simplified in (d) and (e). Finally, bubbles emerge as some wrinkles thicken at the expense of other wrinkles (f). The color map represent the out-of-plane displacement relative to the equilibrium separation $h_{0}$.

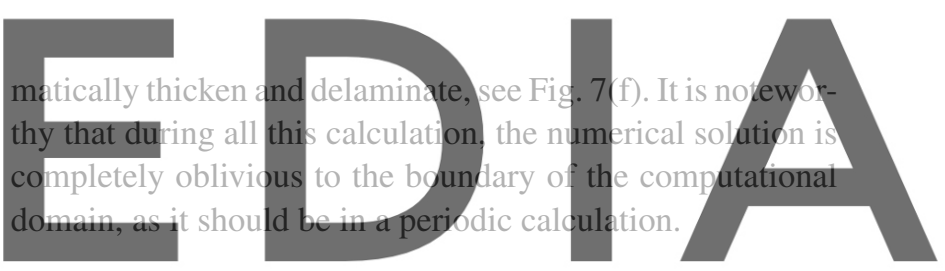

\section{dermilard the version without the watermark}

We have provided simple expressions for the volume enclosed by a material periodic surface and its variation. We have focused on tubular periodic surfaces and doubly periodic surfaces. Even though the boundaries of periodic systems are immaterial, the correct expressions include integrals not only of the surfaces themselves, but also on the boundary curves and a corner contribution in the case of doubly periodic surfaces. We demonstrate the proposed formulation with numerical examples. We motivate this work in our investigations of pressurized carbon nanotubes and graphene sheets, but its applicability is broad.

\section{References}

1. Ahmetoglu, M., Altan, T.: Tube hydroforming: state-of-the-art and future trends. Journal of Materials Processing Technology 98(1), 25-33 (2000)

2. Aleksandrov, V.: Sabitov's conjecture that volume is stationary under infinitesimal bending of a surface. Siberian Mathematical Journal 30(5), 678-684 (1989)

3. Arroyo, M., Belytschko, T.: Finite element methods for the nonlinear mechanics of crystalline sheets and nanotubes. International 
Journal for Numerical Methods in Engineering 59(3), 419-456 (2004)

4. Belytschko, T., Liu, W., Moran, B.: Nonlinear Finite Elements for Continua and Structures. John Wiley \& Sons, England (2001)

5. Bertoldi, K., Reis, P.M., Willshaw, S., Mullin, T.: Negative poisson's ratio behavior induced by an elastic instability. Advanced Materials 22(3), 361-366 (2010)

6. Boddeti, N.G., Liu, X., Long, R., Xiao, J., Bunch, J.S., Dunn, M.L.: Graphene blisters with switchable shapes controlled by pressure and adhesion. Nano Letters 13(12), 6216-6221 (2013)

7. Campelo, F., Allain, J.M., Amar, M.B.: Periodic lipidic membrane tubes. Europhysics Letters 77(3), 38,006 (2007)

8. Cirak, F., Ortiz, M., Schröder, P.: Subdivision surfaces: a new paradigm for thin-shell finite-element analysis. International Journal for Numerical Methods in Engineering 47(12), 2039-2072 (2000)

9. Deserno, M.: Notes on differential geometry (2004). Unpublished

10. Feng, F., Klug, W.S.: Finite element modeling of lipid bilayer membranes. Journal of Computational Physics 220(1), 394-408 (2006)

11. Geers, M., Kouznetsova, V., Brekelmans, W.: Multi-scale computational homogenization: Trends and challenges. Journal of Computational and Applied Mathematics 234(7), 2175-2182 (2010)

12. Koenig, S.P., Boddeti, N.G., Dunn, M.L., Bunch, J.S.: Ultrastrong adhesion of graphene membranes. Nat Nano 6, 543-546 (2011)

3. Levy, N., Burke, S.A., Meaker, K.L., Panlasigui, M., Zettl, A., Guinea, F., Neto, A.H.C., Crommie, M.F.: Strain-induced pseudo-magnetic fields greater than 300 tesla in graphene nanobubbles. Science 329(5991), 544-547 (2010). DOI 10.1126/science.1191700

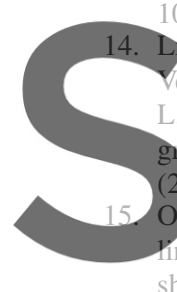

Velamakanni, A., Jung.1., Tutuc, L., Ruoff, R.S.: Large-area synthes graphene films on copper foils. 2009) ervelde, J., Shan, S., Bertoldi, shape. Advanced Materials 24(17), 2337-2342 (2012)
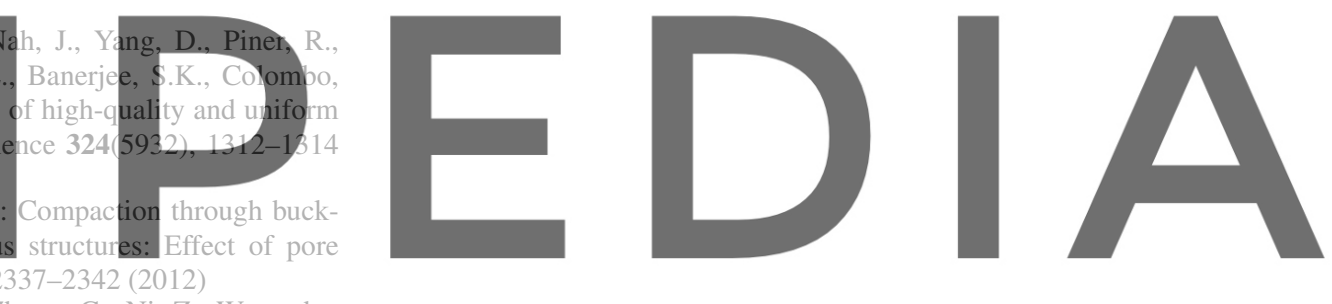

16. Pan, W., Xiao, J., Zhu, J., Yu, C., Zhang, G., Ni, Z., Watanabe,

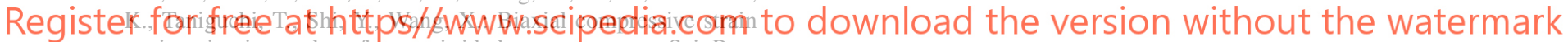
engineering in graphene/boron nitride heterostructures. Sci. Rep. 2, 893 (2012)

17. Robertson, A.W., Bachmatiuk, A., Wu, Y.A., Schäffel, F., Büchner, B., Rümmeli, M.H., Warner, J.H.: Structural distortions in few-layer graphene creases. ACS Nano 5(12), 9984-9991 (2011)

18. Rumpel, T., Schweizerhof, K.: Volume-dependent pressure loading and its influence on the stability of structures. International Journal for Numerical Methods in Engineering 56, 211-238 (2003)

19. Schweizerhof, K., Ramm, E.: Displacement dependent pressure loads in nonlinear finite element analyses. Computers \& Structures 18(6), 1099-1114 (1984)

20. Shima, H., Ghosh, S., Arroyo, M., Iiboshi, K., Sato, M.: Thinshell theory based analysis of radially pressurized multiwall carbon nanotubes. Computational Materials Science 52(1), 90-94 (2012)

21. Staykova, M., Arroyo, M., Rahimi, M., Stone, H.A.: Confined bilayers passively regulate shape and stress. Phys. Rev. Lett. 110, 028,101 (2013)

22. Taylor, N., Gan, A.B.: Submarine pipeline buckling imperfection studies. Thin-Walled Structures 4(4), 295-323 (1986)

23. Zhang, K., Arroyo, M.: Adhesion and friction control localized folding in supported graphene. Journal of Applied Physics 113(19), 193,501 (2013)

24. Zhang, K., Arroyo, M.: Understanding and strain-engineering wrinkle networks in supported graphene through simulations. Journal of the Mechanics and Physics of Solids 72, 61-74 (2014) 